\title{
Measurement and QCD Interpretation of the Deep Inelastic ep Scattering Cross Section by $\mathbf{H 1}$
}

\author{
Rainer Wallny * $\dagger$ \\ CERN, CH-1211 Geneva 23, Switzerland \\ E-mail: irainer.wallny@cern.ch'
}

ABstRACT: Recent H1 cross section measurements at low $x$ and $Q^{2}$ are reported as well as analysis results regarding the behaviour of the proton structure function $F_{2}\left(x, Q^{2}\right)$ and of the longitudinal proton structure function $F_{L}\left(x, Q^{2}\right)$ at low $x$ as well as the measurement of the gluon distribution and of the strong coupling constant $\alpha_{s}$.

\section{Data Sets}

The H1 collaboration recently reported precision measurements [1] inelastic $e p$ scattering cross section in the kinematic range $1.5 \leq Q^{2} \leq 150 \mathrm{GeV}^{2}$ and $3 \cdot 10^{-5} \leq x \leq 0.2$.

The data were recorded with the H1 detector at HERA in 1996 and 1997, and correspond to an integrated luminosity of $20 \mathrm{pb}^{-1}$. The total uncertainty in the "bulk" region of this data is $3-5 \%$ for $Q^{2} \simeq 5-$ $50 \mathrm{GeV}^{2}$. This level of precision is now comparable to the precision of the fixed target measurements. New, preliminary results based on $3 \mathrm{pb}^{-1}$ of data taken in 1999 with increased proton beam energy $E_{p}=$ $920 \mathrm{GeV}$ and improved instrumentation in the backward region of the $\mathrm{H} 1$ detector yield even more precise measurements at low $1.5<Q^{2}<12 \mathrm{GeV}^{2}$ and low $2 \cdot 10^{-5} \leq$ $x<0.07$ and extend the phase space to-

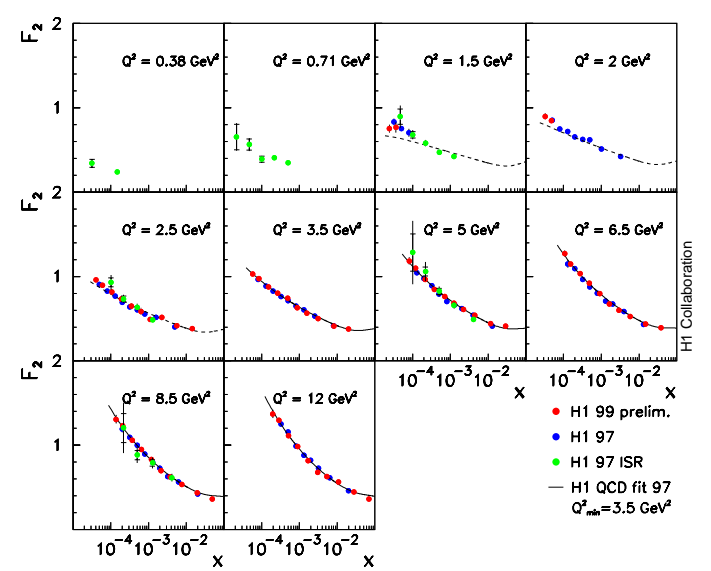

Figure 1: The structure function $F_{2}$ versus $x$ in the low $Q^{2} \leq 12 \mathrm{GeV}^{2}$ region. wards high $y \simeq 0.89$ allowing the longitudinal structure function $F_{L}$ to be accessed at low $Q^{2}$ in a widened $x$ range. Further preliminary results based on 1997 data with an initial ${ }^{*}$ Speaker.

${ }^{\dagger}$ on behalf of the H1 Collaboration 
state radiation photon tagged in the luminosity system of $\mathrm{H} 1$ allow to explore the transition to photoproduction down to values of $Q^{2} \simeq 0.38 \mathrm{GeV}^{2}$, complementing earlier published

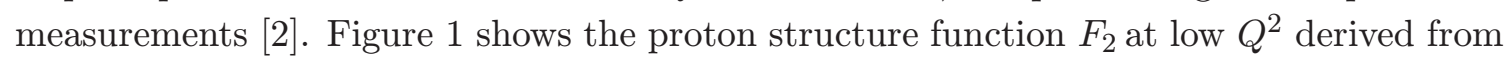
these datasets.

\section{DIS cross section measurement}

The inclusive DIS reduced cross section [i] $\left[\begin{array}{l}1 \\ 1\end{array}\right]$ for $Q^{2}<<M_{Z}^{2}$ is dominated by one photon exchange and is defined as $\sigma_{r}=F_{2}\left(x, Q^{2}\right)-\frac{y^{2}}{Y_{+}} \cdot F_{L}\left(x, Q^{2}\right)$ with $Y_{+}=1+(1-y)^{2}$.

The cross section $\sigma_{r}$ is given by the two proton structure functions $F_{2}$ and $F_{L}$ which are related by $0 \leq F_{L} \leq F_{2}$. Thus the contribution of $F_{L}$ is suppressed in most of the kinematic range explored by the HERA experiments and approximately the relation $\sigma_{r}=F_{2}$ holds, with the exception of the high $y$ region. The event kinematics can be reconstructed from the measurements of the energies and angles both of the scattered positron and of the hadronic final state. This overconstraint contributes to the control of the experimental systematic effects at low $x$ which dominate the total measurement uncertainty. The accessible phase space towards low $Q^{2}$ can be extended by using DIS events in which a photon is radiated collinear to the incident electron, effectively lowering the center of mass energy of the initial state. These events can be identified by detection of the radiated photon in the small angle photon

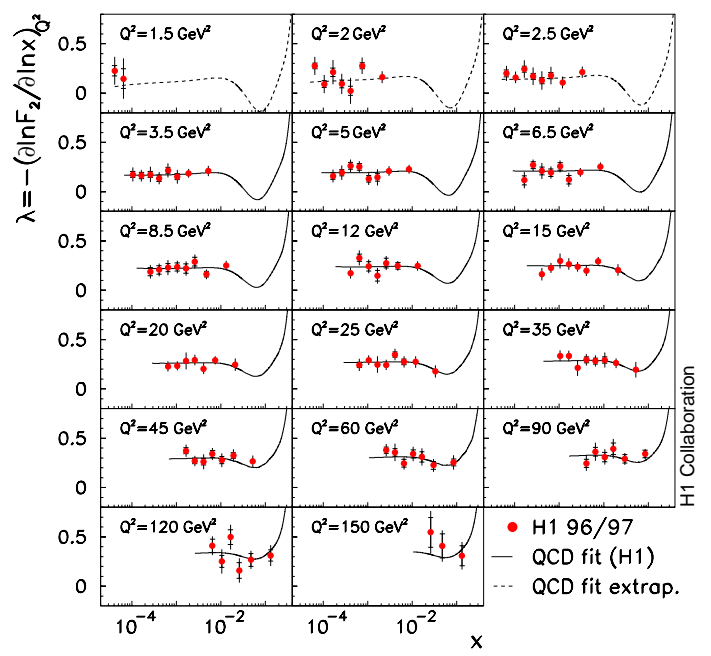

Figure 2: Measurement of the function $\lambda\left(x, Q^{2}\right)$. The inner error bars represent the statistical uncertainty, the full error bars statistical and systematic uncertainties in quadrature. The solid line denotes the NLO QCD fit to H1 data, the dashed line the extrapolation of this fit below $Q^{2}=3.5 \mathrm{GeV}^{2}$. detector of the luminosity system.

\section{The Rise of $F_{2}$}

The proton structure function $F_{2}$ exhibits a strong rise towards low $x$, see figure since $F_{2}$ is related to a cross section and thus is bounded by unitarity, the rise of $F_{2}$ is expected to be eventually damped. A measurement of the rise of $F_{2}$ can be obtained by evaluating the partial derivative $\left(\partial F_{2} / \partial \ln x\right)_{Q^{2}} \equiv-\lambda\left(x, Q^{2}\right)$. Data points at adjacent values of $x$ and fixed $Q^{2}$ are used taking the error correlations into account. As can be seen in in figure rising behaviour of $F_{2}$ is consistently described by a power law, $F_{2} \propto x^{-\lambda}$ for fixed $Q^{2}$. Such a behaviour is expected by approximative solutions of the DGLAP equations [3] in

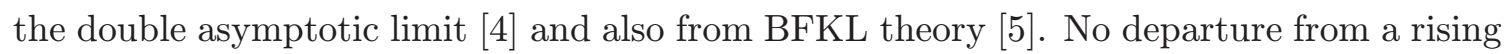


behaviour is visible and the observation of a possible $x$ dependence of $\lambda$ requires higher experimental accuracy.

\section{Extraction of $F_{L}\left(x, Q^{2}\right)$}

The longitudinal structure function $F_{L}$ can reliably be accessed at low $Q^{2}$ by evaluation of the partial derivative of the reduced cross section taken at fixed $Q^{2}$ [i $\left.{ }^{2}\right]$, which is given by

$$
\left(\frac{\partial \sigma_{r}}{\partial \ln y}\right)_{Q^{2}}=\left(\frac{\partial F_{2}}{\partial \ln y}\right)_{Q^{2}}-F_{L} \cdot 2 y^{2} \cdot \frac{2-y}{Y_{+}^{2}}-\frac{\partial F_{L}}{\partial \ln y} \cdot \frac{y^{2}}{Y_{+}}
$$

The new data points on $F_{L}\left(x, Q^{2}\right)$ derived from the preliminary 1999 cross section measurements [i]i] with increased proton energy extend to a lower range in $x$ and are consistent with previous results [i]

\section{QCD Analysis Procedure and the Gluon Distribution}

The DGLAP evolution equations relate the logarithmic $Q^{2}$ derivative of $F_{2}\left(x, Q^{2}\right)$ to the parton distribution functions. At low $x$ (see figure $\overline{\beta_{1}} \mathrm{a}$ )), the scaling violations of $F_{2}\left(x, Q^{2}\right)$ are dominated by the singlet dynamics of sea quarks and gluons and are found to be positive.
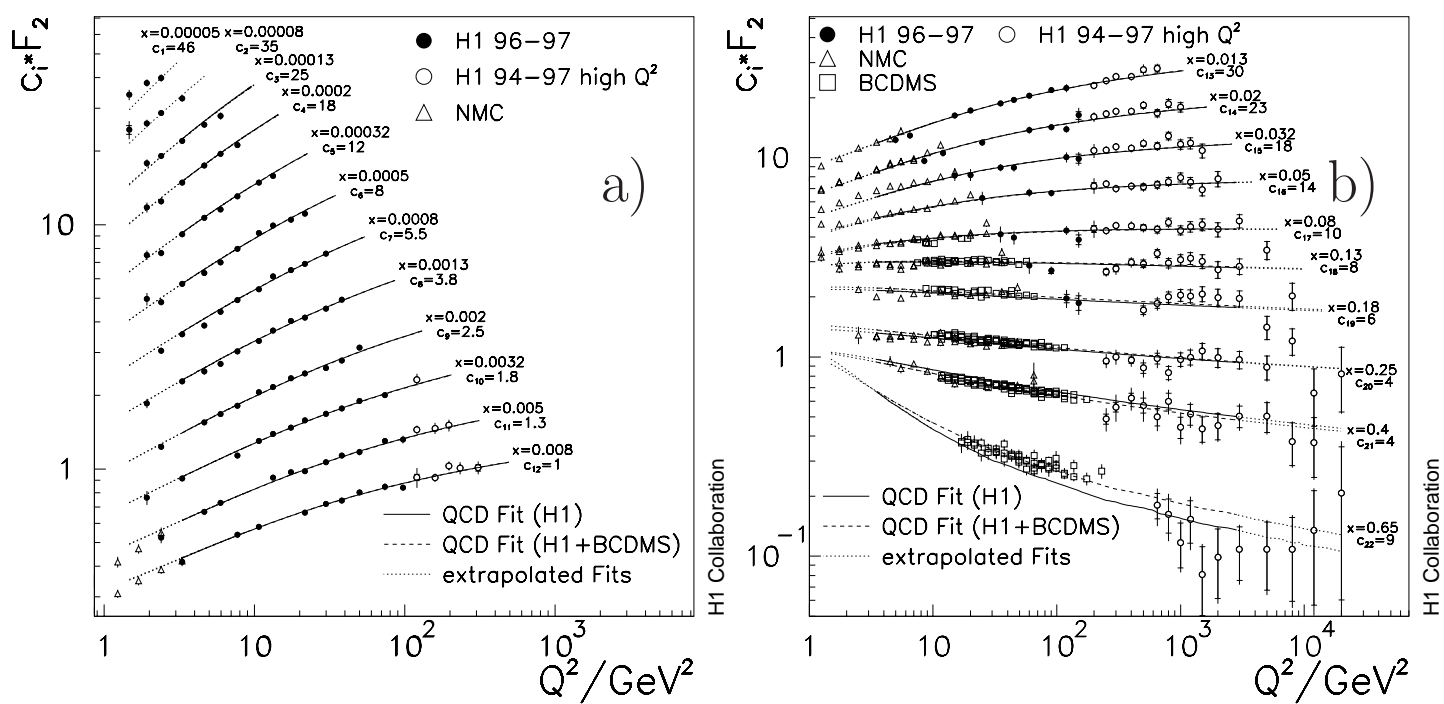

Figure 3: The structure function $F_{2}\left(x, Q^{2}\right)$ for fixed $x$. At low $x<0.01$ (a), the available data is completely dominated by the published $\mathrm{H} 1$ data. At high $x$ (b), the compatibility of the H1 measurements with fixed target data (NMC, BCDMS) is demonstrated. The $Q^{2}$ dependence is compared to a NLO QCD fit to the H1 data (solid line) and to H1 and BCDMS data (dashed line). The dotted curves show the extrapolation of this fit below $Q_{\min }^{2}=3.5 \mathrm{GeV}^{2}$.

In a LO picture [is] $]$, $\left(\partial F_{2} / \partial \ln Q^{2}\right)_{x}$ at low $x$ is roughly proportional to $\alpha_{s} \cdot x g$ for $Q^{2}>3 \mathrm{GeV}^{2}$ and is thus related to a strongly rising gluon, as is demonstrated in figure At higher $x$ (see figure 
quarks which gives rise to negative scaling violations of $F_{2}\left(x, Q^{2}\right)$. Since the singlet and non-singlet sectors are interrelated by the momentum sum rule, precision data at high $x$ and low $x$ are needed to determine the parton distribution functions in the proton.

A NLO DGLAP analysis is performed on published H1 data alone as well as in combination with BCDMS data. The massive heavy flavour scheme is adopted where $u d s$ quarks are evolved and heavy quark contributions to $F_{2}\left(x, Q^{2}\right)$ are added using photon gluon fusion. A cut of $Q^{2} \leq$ $3000 \mathrm{GeV}^{2}$ is imposed to avoid electroweak corrections. A $\chi^{2}$ minimisation is performed to the reduced cross section, taking into account the point-to-point correlated systematic errors of the data in a statistically correct procedure [īi] $\chi^{2}$ retains its statistical interpretation.

A salient feature of this QCD analysis is a novel flavour decomposition of $F_{2}\left(x, Q^{2}\right)$ such that lepton-proton target data can be fitted alone. Thus, uncertainties due to nuclear binding corrections can be avoided. The two quark distributions necessary to describe singlet and non-singlet evolution are chosen such that the quark counting and the momentum sum rules can be imposed and account can be taken of the measured asymmetry of the proton sea as well. Us-

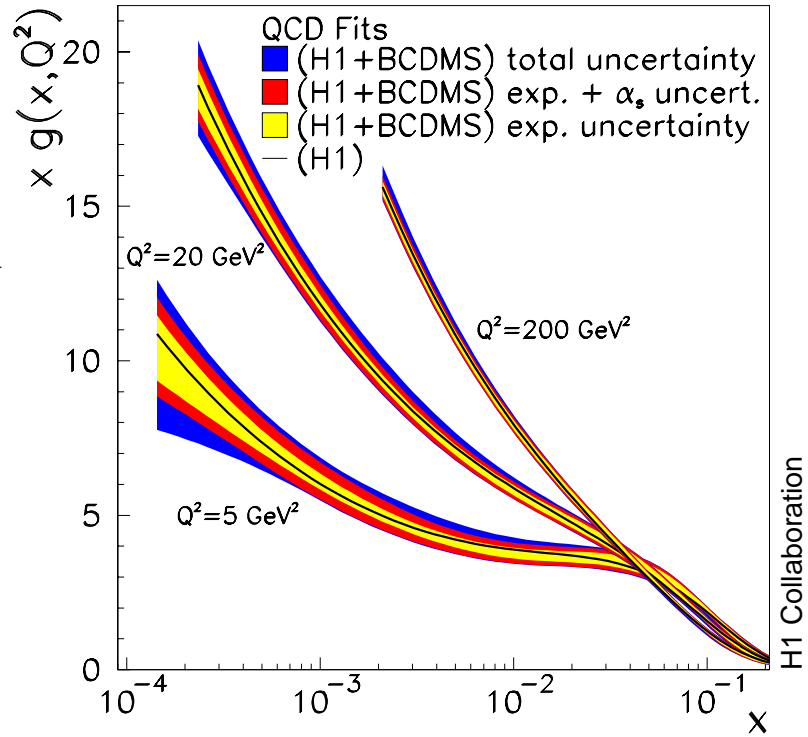

Figure 4: Gluon distribution resulting from the NLO DGLAP QCD fit to H1 ep and BCDMS $\mu p$ cross section data in the massive heavy flavour scheme. The innermost error bands represent the experimental error for fixed $\alpha_{s}\left(M_{Z}^{2}\right)=0.1150$. The middle error bands include in addition the contribution due to the simultaneous fit of $\alpha_{s}$. The outer error bands also include the uncertainties related to the QCD model and data range. The solid lines inside the error band represent the gluon distribution obtained in the fit to the $\mathrm{H} 1$ data alone. ing the criterion of $\chi^{2}$ saturation, the optimal parameterisations of the parton distributions are determined.

A NLO DGLAP fit to precision low $x$ H1 data and previous H1 measurements at high $Q^{2}[\overline{1} \overline{0} \overline{0}]$ determine the gluon distribution to an experimental accuracy of about about $3 \%$ in the range $3 \cdot 10^{-4} \leq x \leq 0.1$ for $Q^{2}=20 \mathrm{GeV}^{2}$, see figure 勇. The model uncertainties on the gluon distribution are dominated by the uncertainties on the charm mass as well as the variation of the cut on the data at lowest $Q^{2}$ to be included in the fit.

\section{Determination of $\alpha_{s}$}

In a combined fit to precision H1 low $x$ and high precision BCDMS high $x$ data, the correlation of $\alpha_{s}$ and $x g$ is resolved yielding $\alpha_{s}\left(M_{Z}^{2}\right)=0.1150 \pm 0.0017(\text { exp })_{-0.0005}^{+0.0009}$ (model). By a rather ad hoc convention, the theoretical uncertainties due to the renormalisation and factorisation scales are estimated by varying the scales between $Q^{2} / 4$ and $4 Q^{2}$. This 
determines an additional theoretical uncertainty on $\alpha_{s}\left(M_{Z}^{2}\right)$ of about \pm 0.005 with the renormalisation scale being the dominant contribution. These results on $\alpha_{s}$ are cross checked with a conventional analysis based on lepton-proton and lepton-deuteron target data yielding the consistent result of $\alpha_{s}\left(M_{Z}^{2}\right)=0.1158 \pm 0.0016(\exp )$. The result obtained by H1 is independent of the choice of the minimum $Q^{2}$ of data included in the fit, i.e. possible departures of NLO QCD in the low $x$ region are not observed. A detailed study of systematic effects reveals that the value of $\alpha_{s}$ favoured by the BCDMS data is larger than hitherto believed [i] $\left.\overline{1}_{-1}\right]$ if the region of largest systematic uncertainty is excluded from the analysis which furthermore is found to be at variance with the SLAC data. This results in a remarkable consistency of the partial contributions of the H1 and BCDMS data to the $\chi^{2}$. If only $\mathrm{H} 1$ data are fitted, the strong coupling constant is determined to be $\alpha_{s}\left(M_{Z}^{2}\right)=0.115 \pm 0.005(\exp )$. This exploratory determination based entirely on H1 data will be improved if higher precision $\mathrm{H} 1$ data become available. Imminent calculations at NNLO [1, $12,1,13$ ind will reduce the theoretical uncertainties by roughly a factor of two. The result obtained is consistent with recent analyses of DIS data which already include con-

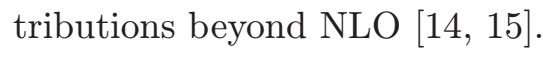

\section{References}

[1] C. Adloff et al., EEur. Phys.

[2] C. Adloff et al., 'Nucl. Phys. B $\mathbf{4} 70(1997) 3$.

[3] Yu. L. Dokshitzer, S'Sov.Phys. JETP 46 (1977) 641 ; V. N. Gribov and L. N. Lipatov, Sov. J. Nucl. Phys. $15(1972) 438$ and 675 G. Altarelli and G. Parisi, 'Nucl. Phys. B 126 (1977) 2988'.

[4] A. De Rujula, S. L. Glashow, H. D. Politzer, S. B. Treiman, F. Wilczek and A. Z ee, Phys. Rev. D 10 (1974) 1649;

R. D. Ball and S. Forte, Phys. Lett. B 335 (1994) 77 [hep-ph/9405320].

[5] E. A. Kuraev, L. N. Lipatov and V. S. Fadin, Sov. Phys. JETP 44 (1976) 443;

E. A. Kuraev, L. N. Lipatov and V. S. Fadin, Sov. Phys. JETP 45 (1977) 199;

Y. Y. Balitsky and L. N. Lipatov, Sov. Journ. Nucl. Phys. 28 (1978) 822.

[6] H1 Collaboration, abstract 799, submitted to this conference.

[7] D.Eckstein, proceedings of DIS2001, Bologna (2001).

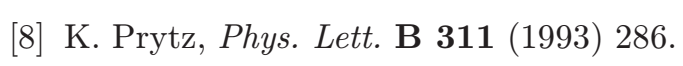

[9] C. Pascaud and F. Zomer, LAL preprint LAL/95-05 (1995). For a recent review, see M.Botje, hep-ph/0110123

[10] C. Adloff et al., 'Eur. Phys. J. 13 - 12000$) 609$ '

[11] A. Milsztaijn and M. Virchaux, 'P̄hys. Lett. B $\mathbf{2} \overline{7} \overline{4}(19 \overline{9} 2) 2211$.

[12] W.L. van Neerven and A. Vogt, hep-ph/0107194.

[13] S.Moch et al., these proceedings.

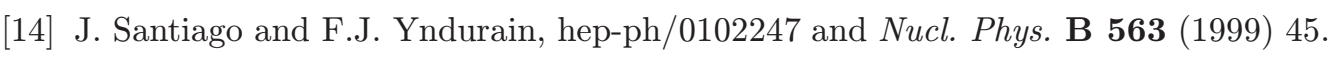

[15] S.I. Alekhin, hep-ph/0107197 and 'Phys. Rev. D $6 \overline{3}(\overline{0} \overline{0} \overline{1}) 0940 \overline{2} \overline{2}$. 